\title{
An exploration of consumer barriers to healthy portion control on the island of Ireland
}

\author{
M. Spence ${ }^{1}$, M. Dean ${ }^{1}$, L. K. Pourshahidi ${ }^{2}$, S. O’Brien ${ }^{3}$ and M. B. E. Livingstone ${ }^{2}$ \\ ${ }^{1}$ Institute of Agri-Food \& Land Use, Queen's University Belfast, Belfast BT9 5AG, UK, ${ }^{2}$ Northern Ireland Centre for Food \\ and Health (NICHE), University of Ulster, Coleraine BT52 ISA, UK and ${ }^{3}$ UCD Institute of Food and Health, \\ University College Dublin, Belfield, Dublin 4, Republic of Ireland
}

\begin{abstract}
As the norm among adults on the island of Ireland (IoI) is to be overweight or obese ${ }^{(1)}$, there is a clear need to provide advice to the general public - not only on healthy food choices, but also on appropriate portion control. However, on the IoI there is a paucity of research on consumer understanding and behavior around food portion size to inform the development of such advice. The primary aim of the current study was, therefore, to qualitatively explore potential barriers to healthy portion control among consumers on the IoI.

Ten focus groups of four to nine participants were formed with a total of 66 members (29 males; 37 females) from the general public. Participants were living on the IoI, and were aged between 18-65 years. The semi-structured discussions elicited participants' perceptions of portion size guidance, and, explored the influence of personal and environmental factors on food portion size consumption. Audiotapes of the discussions were professionally transcribed verbatim, loaded into NVIVO, and analyzed using an inductive, thematic analysis ${ }^{(2)}$.

Qualitative analysis resulted in the identification of eight barrier themes: clarity and relevance of portion size advice; habits ingrained from childhood; product package sizes; value for money; distracted dining; socially acceptable portions; guilt-free portions; and, physical and psychological eating rewards. Participants were unanimous in agreeing that suggested portion sizes were "too small" and "open to interpretation", and described barriers, such as, competitive eating and childhood habits, to healthy portion control. The table outlines example quotes for each theme.
\end{abstract}

\begin{tabular}{|c|c|}
\hline Barrier themes & Example quote \\
\hline Clarity and relevance of portion size advice & "30 g of cereal ... that's like for a guinea pig!" \\
\hline Habits ingrained from childhood & "And if you didn't clear your plate, there was always some poor person ended up in Africa, gonna be hungry!" \\
\hline Product package sizes & $\begin{array}{l}\text { "I live on my own so a lot of things that you'd buy are designed for two people. And then, you'd end up } \\
\text { eating three-quarters of it ..." }\end{array}$ \\
\hline Value for money & "The large popcorn is only another 25 pence or something and you're like, gosh, why not! Portions not an issue!' \\
\hline Distracted dining & "If I'm watching TV...I look down and my plates empty!" \\
\hline Socially acceptable portions & "And then there's a man aspect comes in...'finish that you fairy'!" \\
\hline Guilt-free portions & "For healthy food, you'll just eat away at it cos you know it's fine" \\
\hline Physical and psychological eating rewards & "Give me the chips and dip ... I need to cheer myself up!" \\
\hline
\end{tabular}

Addressing the aforementioned barriers is critical to the development of maximally effective portion control strategies which assist consumers to easily monitor and regulate the food portion sizes they consume.

This material is based upon works supported by safefood, The Food Safety Promotion Board, under Grant No. 07-2010.

1. Ward M, McGee H, Morgan K et al. (2009) Department of Health and Children. Dublin: The Stationary Office.

2. Braun V \& Clarke V (2006) Qual Res Psychol 3, 77-101. 